\title{
Sagnac effect - experimental confirmation of the Theory of Phase Medium
}

\author{
Igor Maydykovskiy, Petras Užpelkis
}

«Biveja» JSC, Private Research Laboratory (Lithuania)

www.biveja.com e-mail: biveja@gmail.com

\begin{abstract}
The article presents a physical model based on the assertion that the space around us is not a emptiness, but represents a homogeneous elastic structure or phase medium. It is proposed to consider a unit quantum resonator to be an element of a phase medium, which makes it possible to extend the principles of quantum mechanics to the theory of a phase medium. Thus, within the framework of the proposed physical model, quantum theory provides an instrument that allows one to consider the transfer of energy of the angular momentum as a process of elastic deformation of the elements of the medium. Understanding the physical mechanism and nature of quantization of the process of propagation of a light wave allows us to ultimately justify the Sagnac interferometer formula.
\end{abstract}

Key Words: Sagnac effect, phase medium, space structure, quantum resonator.

\section{The Sagnac effect}

In 1887, Albert A. Michelson and Edward W. Morley conducted an experiment known as the Michelson-Morley experiment. The purpose of the experiments was to attempt to detect ether. Contrary to expectations, in the experiment - as well as in its later and more precise modifications carried out to date - the result was negative. In his first article on the theory of relativity (1907), Einstein mentions Michelson's failed attempt to detect ether and further puts forward the results of these experiments as the first experimental confirmation of the theory of relativity.

At the end of the 19th, beginning of the 20th century the a ether was considered as the absolute frame of reference, especially in relation to which Maxwell's equations (dating from 1862) had to be written. The Michelson-Morley experiment was not the only one in attempts to detect luminiferous ether; another attempt was made by Georges Sagnac in 1913.

Georges Sagnac changed the design of the interferometer and the experimental scheme. As a result of his experiments with a rotating ring interferometer in 1913, Georges Sagnac discovered the effect, which was later named after him [1]. And although Sagnac himself believed that the result confirms the existence of ether, the scientific community was inclined to believe that the Sagnac effect is a direct confirmation of Einstein's Special Theory of Relativity (STR). Over time, this opinion became an official point of view, although the proposed relativistic principle alone does not explain the physical mechanism of this effect.

As is known, the Sagnac effect consists in the fact that in a rotating ring interferometer, counterpropagating waves acquire a mutual phase shift, which is proportional to the angular velocity of rotation and inversely proportional to the radiation wavelength. This effect, in addition to the optical frequency range, is recorded in the X-ray and radio wave ranges, as well as in other frequency ranges including interference of surface acoustic waves. 
Disputes between proponents of the official version and proponents of explaining the effect by the presence of ether have continued for the past 100 years. However, a general opinion on the nature of this effect has not yet been formed, despite in-depth studies by many famous scientists [2].

An example of this kind of research is the study conducted by Gianni Pascoli of the University of Picardy Jules Verne [3]. Referring to the work of Paul Langevin (1921), he, like many predecessors before him, came to the conclusion that the official version is correct, which claims that this effect is associated with the "relativistic law of velocity addition", which was formulated by Einstein.

At the same time, Gianni Pascoli himself refers to an experiment conducted by Michelson and Gale in 1925 [4]. Michelson and Gale repeated the Sagnac experiment, taking this time the Earth as a spinning disk (as Sagnac himself proposed to do). These authors observed a shift of the bands, similar to the way Sagnac did in his own experiment. The paradox of the situation with the Michelson and Gale experiment is that both proponents of the theory of relativity of Einstein and those who advocate the existence of the ether try to use the positive result of this experiment.

As we know, Sagnac did not recognize Einstein's theory of relativity and considered ether, in the form of some fixed substance, as the structural basis of Space; that is, in the view of Sagnac, ether was a kind of Absolute Reference System. In this sense, the original formula (1-1) operates with the concept of mutual phase shift, which in turn assumes the existence of a reference point that is not associated with a rotating platform. According to the formula, the magnitude of the mutual phase shift is proportional to the angular velocity of rotation and inversely proportional to the wavelength:

$$
\Delta \varphi=2 \cdot \Delta \varphi^{\prime}=\frac{8 \pi^{2} R^{2} \cdot \omega}{\lambda \cdot c}=\frac{8 \pi \cdot A \cdot \omega}{\lambda \cdot c}
$$

here, $\Delta \varphi$ - is the mutual phase shift of the oncoming waves of light; $\Delta \varphi^{\prime}$ - is the phase shift in each channel; $\boldsymbol{\lambda}$ - is the wavelength; $\boldsymbol{R}$ - is the radius of the interferometer; $\boldsymbol{A}$ - is the area covered by the interferometer ring; $\boldsymbol{\omega}$ - is the angular velocity of rotation of the platform; $\boldsymbol{c}$ - is the velocity of light.

That is, as in any interferometer, the phase shift is a necessary and sufficient variable parameter that forms the interference pattern on the recorder screen. Moreover, such an important parameter for the interferometer as its sensitivity will depend on the area of the contour $(A)$ covered by the ring of the interferometer. Here the physical model, supposedly explaining the effect, is built on the assumption that the oncoming light rays move relative to the motionless ether along with the radiation source.

It should be emphasized that the theory of ether since the time of Sagnac has not become a more progressive theory. And just as 100 years ago it is incorrect to consider the explanation of the effect proposed by Sagnac as correct. As an argument for such a conclusion, we can cite the obvious facts of violation by this theory of several fundamental physical principles, namely: the causality principle, the principle of uniformity of the medium, and the principle of quantization. And most importantly, ether cannot be an elastic medium, since according to this theory, space should be an absolute Emptiness in which particles of ether exist and move. 
Comparing the original Sagnac formula and its modern spelling, it is impossible not to notice that, through the efforts of relativists, the Sagnac formula lost any connection with the physical model. Accordingly, using the theory of relativity, it is also impossible to explain the physical mechanism by which supposedly a deceleration or acceleration of the wave front occurs.

According to the relativistic interpretation of the Sagnac formula, it is proposed to calculate not the phase shift, but the difference in the propagation time of counterpropagating waves inside the ring resonator relative to the laboratory reference frame. The proposed rationale is formally described by the formula:

$$
\Delta t=\frac{l}{c-v}-\frac{l}{c+v}=\frac{4 \pi R^{2} \cdot \omega}{c^{2}}=\frac{4 A \cdot \omega}{c^{2}} ;
$$

here, $\boldsymbol{\Delta t} \boldsymbol{t}$ - is the path difference between the fronts of the oncoming waves;

$v$ - is the linear velocity of the ring resonator;

$\boldsymbol{l}=2 \pi R$ - is the length of the propagation path of the wave front.

Using the theory of relativity as an evidence base for explaining the Sagnac effect, supporters of this hypothesis make one fatal mistake. It follows from the above formula that the velocity of propagation of the wave front can be greater than the velocity of light $\left(\boldsymbol{c}+\boldsymbol{v}^{\prime}\right)$, which is fundamentally impossible in the material World! And what, incidentally, is pointed out by many authors who criticize the attempt to explain the Sagnac effect by the action of the "relativistic law of velocity addition", considering this law itself as a kind of abstraction.

Moreover, the main mistake of the relativistic approach is that the laboratory inertial reference system is proposed as a measuring system. However, according to the experimental design, the radiation source and the light channel (resonator) belong to a rotating platform, that is, a noninertial reference frame. Accordingly, all measurements must be made with respect to the rotating platform itself. Thus, there can be no talk of any relativistic addition of velocities! After all, an observer associated with a non-inertial system does not know that the platform is rotating; for the observer connected with the platform, the direct and oncoming rays travel the same path at the velocity of light. The funny thing is that much-respected scientists do not want to admit this obvious fact.

The reason for doubt is added by the fact that the Theory of Relativity itself has recently been constantly criticized. Based on the postulate "On Absolute Emptiness", this theory became a brake on the development of adequate physical models of such fundamental concepts as ENERGY, MATTER and FIELD. Considering the process of energy propagation in empty space, without material intermediaries, this Theory violates the fundamental principle of physics - the principle of causality.

An example is the reluctance of official science to recognize the existence of longitudinal electromagnetic waves. This reluctance can be explained quite simply: the fact is that a longitudinal wave can exist only in a medium that allows compression and tension. Recognition of the existence of such an environment means recognition of the presence of the Absolute Reference Frame, that is, recognition of the presence of a structured phase space. Therefore, with some irony, we can say that the result of the Sagnac experiment is not the only one that really "suffered" from the influence of such postulates. 
It is not superfluous to recall that the Sagnac effect exists, and has already found application in many measuring facilities. The most famous of them are fiber-optic laser gyroscopes. Therefore, our further studies aim to establish a real physical mechanism and propose a model that can explain the results of the experiment and which at the same time satisfies the Sagnac formula.

To understand the true nature of the Sagnac effect, the first thing to do is to change the paradigm and abandon the assertion that the space around us is Emptiness. John Maxwell, the founder of the theory of propagation of electromagnetic waves, argued that, “... no matter what difficulties we face in the future in our attempts to develop a sound idea of the structure of space, there is no doubt about one thing - it cannot be empty. The space is occupied by the material substance or body, the most extensive and, we must think, the most homogeneous that we know".

But first, let's try to understand the physical mechanism of limiting the speed of light. Being a fundamental constant, the value of the velocity of light should reflect the essence of the processes occurring in a phase medium, the inertial properties of which will determine the nature of these processes.

\section{Physical model of the phase Medium}

Within the framework of the new paradigm, space is a homogeneous elastic structure or phase medium. To determine the basic characteristics of an element of the phase Medium, one can start from the understanding that these characteristics are somehow present in world constants. This will not be difficult to do if you imagine that the development of any physical process begins at the level of fine structure, that is, at the level of the phase medium.

A well-known formula that determines the value of the minimum size of matter and which connects three world constants. This formula defines $\lambda_{0}$ as the minimum wavelength corresponding to the minimum size of matter or the quantum of energy:

$$
\lambda_{0}=\sqrt{\frac{2 \pi G \cdot h}{c^{3}}}(\mathrm{~m})
$$

here: $\boldsymbol{h}=6,626069 \cdot 10_{-34}(\mathrm{~J} \cdot \mathrm{sec})$ or $\left(k G \cdot \mathrm{m}^{2} \cdot \mathrm{sec}^{-1}\right)$ - Planck's constant; the main constant of quantum theory - a coefficient relating the quantity of the energy of electromagnetic radiation with its frequency;

$\boldsymbol{G}=6,6719199 \cdot 10_{-11}\left(\mathrm{~m}^{3} \cdot \mathrm{sec}^{-2} \cdot \mathrm{kG}^{-1}\right)$ - is the gravitational constant; $c=2,997924 \cdot 10^{8}(\mathrm{~m} / \mathrm{sec})$ - is the velocity of light.

From this expression, it follows that relative to a quantum of energy (the minimum size of matter), an electromagnetic wave is inversely proportional to a longitudinal (gravitational) wave in accordance with the principle of feedback in a two-dimensional frequency plane.

Formula (2-1) reflects the essence of one of the basic Laws, which allows you to establish the relationship of the electromagnetic shell of a quantum of energy with the internal "core" in the form of a quantum of gravitational energy. This Law speaks of the existence of a continuous process and mechanism that provides a reversible mutual transformation of one type of energy gravitational, into another type - into electromagnetic energy. 
It should be noted that for our model the term "longitudinal waves" is identical to the term "gravitational waves", however, the latter definition cannot be interpreted as recognition of the existence of gravitational waves in their classical interpretation, which is waves generated directly by the mass itself.

Now, substituting the required values of world constants into formula (2-1), we obtain the numerical value of the minimum wavelength corresponding to the size of the energy quantum. As a result of the calculations, the value of the minimum wavelength turns out to be equal:

$$
\lambda_{0}=1,0153421 \cdot 10_{-34}(\mathrm{~m}) \text {; }
$$

What corresponds to the minimum size of matter. At the same time, through the fine structure constant, the energy quantum determines the 2nd harmonic wavelength, which is equal to the radius of the phase medium element $\left(\boldsymbol{r}_{\boldsymbol{m}}\right)$ and which forms its structure:

$$
r_{m}=\frac{\lambda_{0}}{2 \pi \alpha^{-1}}=\lambda_{m(2)}=1,179228 \cdot 10_{-37}(\mathrm{~m})
$$

here, $\alpha=7.2973525698 \cdot 10_{-3} \approx 1 / 137$ is the fine structure constant.

The model of the structural element of the phase medium can be represented as a limited region of space, which can be defined as a single quantum resonator or $\boldsymbol{m}$-cavity. The elasticity of the phase medium is ensured thanks to the constantly operating mechanism of excitation of quantum resonators with the formation of standing spherical longitudinal waves inside these cavities. A spherical standing longitudinal wave is an absolutely rigid "construction" in the structure of the medium element - if such a term can be applied to a wave. The mechanism of excitation of single quantum resonators should be based on a number of properties of the space, which are:

- The presence of longitudinal excitation waves at the resonant frequencies of the medium element;

- Multidimensional symmetry of the space, due to which the transfer of momentum energy in any direction from element to element is lossless;

- The quantum nature of the mechanism of excitation and transmission of the angular momentum energy.

The condition of the continuity of space dictates the only possible form of an element of the medium - this is a rhombic dodecahedron or Wigner-Seitz cell. This statement is based on a single criterion - this is the requirement of the absence of a void in the structure of the phase medium.

The Wigner - Seitz cell has one significant feature, due to which the mechanism of forming cells in the form of $\boldsymbol{m}$-cavities in the space is provided. This feature is that the planes of all 12 congruent faces are perpendicular to the straight lines connecting the center point of the cell with the center of the corresponding edge. It follows from what has been said that during the formation of spherical longitudinal waves inside the $\boldsymbol{m}$-cavity, the center of the cell also remains the center of forces.

Another important property of a Wigner-Seitz cell is its congruence already mentioned, that is, the ability of any cell to be transferred to another similar one by shift, rotation and mirror image or compositions of the indicated actions. The indicated property of the Wigner-Seitz cell supports the mechanism of the formation of any structures from elements of the medium in accordance with the laws of multidimensional symmetry of the space, ensuring the copying and repetition of any fractal spatio-temporal configurations. 
According to the classical theory, the propagation of waves in a homogeneous elastic medium occurs due to bias currents. By analogy - the propagation of the angular momentum in a phase medium can be represented through the phase shift of the fundamental mode in the structure of the medium element.

Accordingly, the process itself can be quantified by the formula:

$$
\boldsymbol{j}_{m}=\frac{e_{m}}{\Delta r_{m}} \cdot\left[\frac{d\left(\Delta r_{m}\right)}{d t}\right]
$$

here: $\Delta \boldsymbol{r}_{\boldsymbol{m}}$ - is the value of the allowable deformation of the medium element;

$\boldsymbol{j}_{\boldsymbol{m}}$ - is the phase shift of the fundamental mode of the medium element;

$\boldsymbol{e}_{\boldsymbol{m}}$ - is the value of the energy potential of an element of the medium.

The structure of the phase medium gives reason to assert that the value of the energy potential $\left(\boldsymbol{e}_{\boldsymbol{m}}\right)$ cannot be determined through the magnitude of the spectral density, in the form of the total energy of oscillations in the structure of the $\boldsymbol{m}$-cavity. The physical meaning will have a model according to which the internal energy of an element of the medium is determined by its prestress. The value of this energy together with the magnitude of the allowable deformation will ultimately determine the elastic properties of the medium.

It should be noted that for an unstructured phase medium, that is, in the absence of synchronization of oscillations between the main modes of $\boldsymbol{m}$-cavities, the specific energy potential of the space will be composed of the potential energy associated with the degree of prestressing of the $\boldsymbol{m}$ cavities, as well as with the degree of their deformation in the structure of longitudinal waves in the process of their formation and propagation.

In the classical paradigm, the total potential energy of deformation of the phase medium, connected with: a) the preliminary stress of the phase medium, and b) the energy of oscillations longitudinal waves in the structures of the material World, - is called "dark energy". Although, for obvious reasons, such a definition of dark energy cannot be found in scientific publications that reflect the official point of view, according to which dark energy is called, "it is not known what". That is, dark energy is presented as a kind of hypothetical form of energy. The fact of the existence of dark energy is confirmed by experimental data, and its value is estimated at up to $75 \%$ of the total energy of the space.

The propagation velocity of the angular momentum energy in a phase medium depends on its inertial properties. Therefore, based on the presented model of the structural element of the phase medium, we can try to bring the concept of inertia in accordance with the physical model. This can be done if you use the law "On the Continuity of the Energy propagation Process", which was formulated by Nikolai Umov.

In the years $1870-1874$, after conducting a series of experiments, Nikolai Umov made the assumption that inertia is a manifestation of the elastic properties of the medium itself and the causality (continuity) of the angular momentum energy transfer process [5]. In other words, Nikolai Umov suggested that the mechanism of inertia is associated with a slowdown in the velocity of propagation of the momentum energy inside the body. That is, due to the "continuity" of the transmission process, the momentum energy is transmitted from element to element inside the body with delay, which manifests itself in the form of inertia. 
The law on the continuity of the energy propagation process can be written in modern mathematical language in the following form:

$$
\frac{\partial U}{\partial t}+\operatorname{div} \vec{S}=0
$$

here, $\vec{S}$ - is the Umov-Poiting vector

It should be noted that this law excludes any thought about the concept of instant long-range action, according to which bodies interact with each other without material intermediaries, through emptiness, at any distance. It is believed that such interaction is carried out with infinitely high velocity. One example of supposedly direct action at a distance is the force of universal gravitation in Newton's classical theory of gravity.

Developing the assumption of Nikolai Umov, it will be logical to say that for our model inertia is not a property of body mass, as the classical theory of Newton claims; Inertia is also not related to the viscosity of the "ether", as proponents of the existence of ether can claim. Inertia is a manifestation of the elastic properties of the elements of a phase medium that are in an excited state and are constituent elements of the body itself.

That is, inertia is associated with the finiteness of the time of synchronization of environmental elements belonging directly to the "body". The body, as it were, every time anew "incarnates" at the speed of propagation of the pulse, that is, at a speed equal to the velocity of light. This process can be represented as the formation of an object on a conditional "monitor" with a frequency of incarnations, each time from new "pixels", that is, from new elements of the environment. In fact, the frequency of incarnations is the frequency of the gamma-quantum for the "red border", which can be calculated based on the law of conservation of energy in the process of incarnation electron:

$$
E=v_{r b} \cdot h=2 m_{e} \cdot c^{2}=1,031 \mathrm{MeV}
$$

here, $\boldsymbol{h}$ - is the Planck's constant, $\boldsymbol{m}_{\boldsymbol{e}}$ - is the rest mass of the electron

Thus, the frequency of the "red border", $v_{r b}=2,4892 \cdot 10^{20}(\mathrm{~Hz})$ will be the frequency of materialization (incarnation) of the electron and therefore of any body. Therefore, speaking of the inertia of the body, we cannot connect this concept with its mass. Accordingly, the value of the mass itself can be represented as a measure that allows, according to the degree of synchronization of vibrations and the degree of deformation of the elements of the phase medium, to compare different levels of modification of the medium, linking these levels with different density of matter. Postulate: Matter is a modified medium in the form of a homogeneous elastic structure, consisting of a certain set of structural elements - quantum resonators ( $\boldsymbol{m}$-cavities) with different degrees of deformation and different degrees of synchronization of oscillations.

The validity of this Postulate follows from the above formula (2-6), according to which the particle energy can be represented through the Planck constant and the oscillation frequency. The Planck's constant corresponds to energy equivalent to a quantum of action that occurs when the oscillation frequency changes by $1 \mathrm{~Hz}$. According to the formula, the total energy of the particle will be equal to the frequency times the Planck's constant. 
Presenting his formula, Planck specified that his "constant" connects the energy of the angular momentum with the spatial frequency, and the action itself with the phase gradient. It should be noted that in quantum electrodynamics, the energy of particles is measured in electronvolts $(\mathrm{eV})$. It is this circumstance and Einstein's formula $\boldsymbol{E}=m_{e} \cdot c^{2}$ that make it possible to identify the rest mass with energy. According to Planck's formula, gamma-quantum, which has no mass, also has energy. For a gamma quantum, energy exists only in the form of oscillation energy, and there is no "rest energy" which Einstein interpreted as mass.

It is also necessary to recall that formula (2-6) reflects the essence of the physical process associated with the birth of two particles - an electron and a positron. This event occurs as a result of the excitation of a phase medium by a gamma-quantum with energy of at least, $E=1,031 \mathrm{MeV}$. That is, a massless particle or just a wave of a certain energy gives rise to two particles having mass.

Equality (2-6) indicates the identity of two different forms of Energy and allows you to connect the energy of matter with a frequency-phase gradient. If both sides of the equation are expressed in terms of normalized increments in the form of a differential equation, then by simple transformations, we can present the final Einstein-Planck formula:

$$
h \cdot \frac{d v}{d t}=c^{2} \cdot \frac{d v / \overrightarrow{d r}}{d t}
$$

here, $d v / d \vec{r}=d \rho$ - is the unit density increment expressed through the spatial phase gradient.

The resulting equality in structure resembles Einstein's formula, $\boldsymbol{E}=\boldsymbol{c}^{2} \cdot \boldsymbol{m}$, and gives us reason to assert that the Planck's constant associates energy with a frequency gradient, and the momentum of action, that is, the manifestation of energy, with a spatial phase gradient, which is identical to an increase in the density of the medium. Thus, this ratio reflects the essence of the model of the physical process that underlies the materialization of the wave matrix, that is, it reveals the "miracle" of the incarnation of matter from the phase medium.

Unlike the natural process, conducting experiments that allow in laboratory conditions to record the fact of the birth of an electron-positron pair from a phase medium is associated with the need to create high gradients of field structures. It can be constant and uniform mutually perpendicular electric and magnetic fields, or strong laser fields. As an example, we can refer to a paper devoted to studies related to the problem of the production of an electron - positron pair $\left(\mathbf{e}^{+} \mathbf{e}^{-}\right)$from vacuum when irradiated in Combined Laser and Coulomb Fields [6].

The conclusion about the wave nature of the processes that form the material world can also be made from an analysis of the Schrödinger equation [7]. As known, the appearance of the Schrödinger equation in 1925 was preceded by de Broglie's revolutionary assumption that not only light but also all material bodies have wave properties. Schrödinger, describing the behavior of a particle by a wave function, identified this concept with a probability wave.

Thus, the classical differential equation of the wave function was transformed into an equation that describes the probability of a particle being at a given point in space:

$$
-\frac{\hbar^{2}}{2} \frac{1}{m} \Delta \Psi+U \cdot \Psi=i \cdot \hbar \cdot \frac{\partial \Psi}{\partial t}
$$

here, $\boldsymbol{\Psi}$ - is the wave function; $\boldsymbol{\Delta}$ - is the Laplace operator; $\boldsymbol{h}$ - Planck's constant;

$\boldsymbol{U}$ - potential energy; $\boldsymbol{m}$ - is the rest mass of the particle; $\boldsymbol{i}=\sqrt{-1}$. 
Obviously, the de Broglie probability wave does not explain the physical mechanism of the process of transformation of the wave into a particle. However, one should not conclude from this that the Schrödinger equation is incorrect. If you expand the wave function, it turns out that it is represented as the product of two functions, one of which determines the position of the particle in space, and the other as an oscillator - depends on time: $\Psi=\Psi(x, y, z) \cdot \varphi(t)$. The Laplace operator $(\Delta)$ is equivalent to sequentially taking the operations of the gradient and divergence: $\Delta=$ div grad. According to the theory of the phase medium, the potential field energy (U) can be identified with the degree of deformation of the structural elements of the phase medium.

Now, if we will not invent a mysterious, unknown form of energy for matter, but represent the mass as the energy equivalent, expressed through a spatial phase gradient, the Schrödinger equation will describe the physical process of the reversible transformation of a wave into "a particle".

$$
\hbar \cdot \frac{c^{2}}{\omega}(\operatorname{div} \mathrm{grad}) \cdot \Psi+i \cdot \hbar \cdot \frac{\partial \Psi}{\partial t}=U \cdot \Psi
$$

This process is characterized by potential energy, which at each moment of time is equal to the sum of the particle energy equivalent to its mass (1st term of the equation) and the energy of the Electromagnetic Longitudinal Wave (2nd term of the equation).

Thus, the quantum nature of the wave propagation process indicates that no transformation of the wave into a particle actually takes place and that, taking into account the quantum nature of the wave process, the fact of registration of a particle is only one of the events of its "incarnation", which was detected using a detector. Naturally, the process of particle registration will be probabilistic in nature, which is confirmed by experimental data, for example, Jung's experiments.

\section{Explanation of the Sagnac effect}

If we are guided simultaneously by the Planck model and the model underlying the Schrödinger equation, then in the framework of the theory of the phase medium, the Sagnac effect can be explained quite simply. Recall the definition of inertia for a phase medium: Inertia is a manifestation of the elastic properties of medium elements that are in an excited state and are constituent elements of the body or process itself. Inertia is inextricably linked with the finiteness of the time of transition to the state of excitation (synchronization) of the elements of the phase medium, that is, with the delay in the materialization of structures and objects in the process their incarnation. Undoubtedly, in our case, such an "object" is a light wave.

As follows from the physical mechanism previously considered, associated with the propagation of an electromagnetic wave, the frequency of incarnation is nothing but the frequency of the gammaquantum for the "red border", that is: $\boldsymbol{v}_{\boldsymbol{e}}=\boldsymbol{v}_{\boldsymbol{r} b}=2,489213 \cdot 10^{20}(\mathrm{~Hz})$. This frequency, based on the energy conservation law (2-6), determines the minimum gamma-quantum energy necessary for the materialization (incarnation) of an electron, $E=1.031 \mathrm{MeV}$, and allows you to calculate the value of the incarnation period:

$$
\tau_{e}=\frac{1}{v_{r b}}=\frac{h}{2 m_{e} c^{2}}=0,40173 \cdot 10_{-20}(\mathrm{sec}) ;
$$

here, $\boldsymbol{h}$ - is Planck's constant; $\boldsymbol{m}_{\boldsymbol{e}}$ - is the rest mass of the electron 
It is not difficult to understand the obvious invariance of the expression. Since this formula allows you to connect not only the energy of the gamma-quantum with its frequency, but also to determine the velocity of light as a derivative of the process of incarnation:

$$
c=\sqrt{\frac{v_{r b} \cdot h}{2 m_{e}}}
$$

That is, the fundamental physical law of the reproduction of matter through of the incarnation mechanism by the feedback principle, reflecting the inertial properties of the phase medium, uniquely determines the value of the velocity of propagation of a light wave equal to $(\boldsymbol{c})$.

Now we can use the conclusions regarding the nature of quantization of the process of propagation of a light wave and consider two modes of the operation of the Sagnac interferometer:

1. The stationary mode of operation will be characterized by the number of incarnation $\left(\boldsymbol{P}_{\text {inc }}\right)$, which will take place per time $(\mathrm{t})$, corresponding to the duration of propagation of the wave front:

$$
t=L / c=2 \pi R / c ;
$$

here, $\boldsymbol{R}$ is the radius of the interferometer

2. When the interferometer rotates with an angular velocity $(\omega)$, the dividing mirror for the specified propagation time $(\boldsymbol{t})$ of the wave front, move to a distance $\left(\Delta L^{\prime}\right)$ :

$$
\Delta L^{\prime}=\frac{L}{c} \cdot \omega R=\frac{2 \pi R \cdot \omega R}{c} ;
$$

The time required by the light wave to overcome this distance will be:

$$
\Delta t^{\prime}=\Delta L^{\prime} / c=\frac{2 \pi R^{2} \cdot \omega}{c^{2}} ;
$$

This time will correspond to a phase shift:

$$
\Delta \varphi^{\prime}=2 \pi \cdot v \cdot \Delta t^{\prime}=2 \pi \cdot v \cdot \frac{2 \pi R^{2} \cdot \omega}{c^{2}} ;
$$

The difference between the wave fronts in the direction and against the direction of rotation of the platform, expressed in the number of incarnation (quanta), will be:

$$
\Delta P_{i n c}=\left(P_{i n c}+\Delta t^{\prime} / \tau_{e}\right)-\left(P_{i n c}-\Delta t^{\prime} / \tau_{e}\right)=2 \Delta t^{\prime} / \tau_{e}
$$

Then the stroke difference in seconds:

$$
\Delta t=\Delta P_{i n c} \cdot \tau_{e}=2 \Delta t^{\prime}=\frac{4 \pi R^{2} \cdot \omega}{c^{2}}=\frac{4 A \cdot \omega}{c^{2}} ; \quad(\mathrm{sec})
$$

What corresponds to the mutual phase shift:

$$
\Delta \varphi=2 \pi \cdot v \cdot \Delta t=\frac{8 \pi \cdot \pi R^{2} \cdot \omega}{\lambda \cdot c}=\frac{8 \pi \cdot A \cdot \omega}{\lambda \cdot c} ; \quad(\mathrm{rad})
$$

here, $\Delta \varphi$ - is the mutual phase shift of the oncoming waves of light; $\boldsymbol{\omega}$ - is the angular velocity of rotation of the platform; $\boldsymbol{A}$ - is the area covered by the interferometer ring; $c$ - is the velocity of light; $\lambda$ - is the wavelength and, $v=\frac{c}{\lambda}$ - is the frequency of the radiation source.

That is, the main variable parameter determining the dynamics of interference fringes at the receiving detector is the difference in the number of incarnations $\left(\Delta P_{i n c}\right)$ of counterpropagating waves. 
The given result exactly corresponds to the Sagnac interferometer formula, and was obtained without involving the "relativistic law of velocity addition", ether in its classical interpretation, and also without other mathematical tricks.

Thus, the Sagnac effect proves the discrete nature of the process of propagation of electromagnetic waves and at the same time is a direct confirmation of the existence of a phase medium and the law of the formation of the material World, based on the continuous process of embodiment of physical reality with a frequency of gamma-quantum $\left(v_{r b}\right)$. This frequency is the main "culprit" in limiting the velocity of light to:

$$
c^{*}=\sqrt{\frac{v_{r b} \cdot h}{2 m_{e}}}=2,997924 \cdot 10^{8}(\mathrm{~m} / \mathrm{sec}) .
$$

That is, in relation to any processes in the material World, the velocity of light is the maximum possible velocity, and the process of light propagation in space does not depend on the velocity of the source, since the "source" sets only the point of initial excitation of the medium. After which the propagation of a wave of light occurs by its reradiation in an elastic medium. Moreover, the velocity of this process will always be limited by the inertial properties of the medium itself, that is, the velocity of the angular momentum transfer. Therefore, it is completely unimportant for the process of propagation of an electromagnetic wave - the radiation source is moving or standing still. In particular, this explains the ability of an electromagnetic wave to propagate in a packet, that is, to exist far from the radiation source.

In this sense, the Sagnac effect confirms the opinion of many scientists that the "relativistic law of velocity addition" is a law that reflects a virtual, abstract model. In fact, all the misconceptions of Einstein, including the Special Theory of Relativity, are related to the fact that he could not understand the reason for limiting the velocity of light. The consequence of which was the need to consider space an emptiness, and the corpuscular Theory became the only possible theory explaining the existence of matter.

\section{Findings}

The Theory of the Phase Medium undoubtedly needs experimental confirmation of the main points. For example, the presence of a wave matrix in the form of longitudinal, standing waves can be confirmed using a specially designed device based on two Sagnac interferometers operating in the differential detection mode. Any experiment conducted that allows one to confirm certain positions of thorium, can lead to the development of new directions in experimental physics. Which in turn will lead to a change in our ideas about the reality surrounding us and will affect many technologies - from energy and transport to medicine and food production.

\section{Conclusion}

The presented simple proof of the Sagnac formula is based on a natural physical mechanism. The basis of this mechanism is the wave structure of space and the quantum nature of all processes, including the process of the formation of matter. This model also allows simple and elegant to substantiate the physical mechanism of any process that underlies the physics of reality. Therefore, the presented work is the first of a series of articles prepared by the authors for publication. 
In subsequent articles, physical models of basic concepts, such as FIELD, ENERGY, SPACE, TIME including a model of the process of forming the structure of space, will be considered. A model of the physical process of propagation of electromagnetic waves and a model of the process of gravitational interaction will also be considered. Based on the Theory of a Phase Medium, the mechanism of gravitational interaction allows us to propose an other planetary Theory, different from the existing. Several articles will be devoted to this topic.

The theory of the phase medium can become the only platform that will allow us to formulate and justify the principles of the Grand Unified Theory (GUT). This will be the final article of the entire cycle of work.

\section{References}

[1] Georges Sagnac: Sur la preuve de la réalité de l'éther lumineux par l'expérience de l'interférographe tournant. // Comptes Rendus Physique, 157: 1410-1413. 22 décembre 1913. (Georges Sagnac: Evidence of the reality of the luminiferous ether in the experiment with the rotation of the interferometer. December 22, 1913).

[2] The Sagnac effect. // From Wikipedia, the free encyclopedia. https://en.wikipedia.org/wiki/Sagnac_effect

[3] Gianni Pascoli: The Sagnac effect and its interpretation by Paul Langevin. // Comptes Rendus Physique. Volume 18, Issues 9-10, November-December 2017, pp. 563-569. https://www.sciencedirect.com/science/article/pii/S1631070517300907

[4] Michelson A. A., Gale H. G.: The Effect of the Earth's Rotation on the Velocity of Light, II. // Astrophysical Journal. ApJ., 61, pp. 140-145, 1925.

[5] Umov N.: Ableitung der Bewegungsgleichungen der Energie in continuirlichen Körpern. // Z. Math 19, pp. 418-431 (217). 1874. (The Derivation of equations of motion of energy in solid bodies).

[6] A. I. Milstein et al.: Polarization-Operator Approach to Electron-Positron Pair Production in Combined Laser and Coulomb Fields. // Physical Review A - Atomic, Molecular, and Optical Physics, vol. 73, no. 6, pp. 062106-1 - 062106-10, American Physical Society (APS), Jun 2006.

[7] Schrödinger E.: The Interpretation of Quantum Mechanics: Dublin Seminars (1949 - 1955) and Other Unpublished Essays. // Woodbridge, Conn.: Ox Bow Press, 1995. 